\title{
Formulation and In vitro Dissolution Characteristics of Sustained-Release Matrix Tablets of Tizanidine Hydrochloride
}

\author{
Ghulam Murtaza ${ }^{1 *}$, Hanif Ullah ${ }^{1}$, Shujaat Ali Khan ${ }^{1}$, Sadullah Mir $^{2}$, Abida \\ Kalsoom Khan ${ }^{2}$, Bushra Nasir ${ }^{3}$, Saira Azhar ${ }^{1}$ and Mubashir Ali Abid ${ }^{1}$ \\ ${ }^{1}$ Department of Pharmacy, ${ }^{2}$ Department of Chemistry, COMSATS Institute of Information Technology, Abbottabad, ${ }^{3}$ Faculty of \\ Pharmacy, Bahauddin Zakariya University, Multan, Pakistan
}

*For correspondence: Email: gmdogar356@gmail.com; Tel: +92-992-383591-5; Fax: +92-99238344

\begin{abstract}
Purpose: To formulate sustained-release (SR) matrix tablets of tizanidine hydrochloride (THC) and to investigate the effect of matrix polymer type on drug release profile of drug.

Methods: Matrix tablets of THC were prepared by direct compression method using a combination of hydroxypropylmethylcellulose (HPMC) and ethylcellulose (EC) in varying ratios. In all the formulations, the amount of THC was $6.87 \mathrm{mg}$ (equivalent to $6 \mathrm{mg}$ base). USP type-I (basket) apparatus was used for the dissolution study. The dissolution study was performed in $0.1 \mathrm{M} \mathrm{HCl}$ for the first $2 \mathrm{~h}$ and in phosphate buffer ( $\mathrm{pH}$ 6.8) for another $10 \mathrm{~h}$. The dissolution data were subjected to drug release models to ascertain the kinetics of drug release. Additionally, in vitro swelling and buoyancy studies were carried out on the optimized formulation. The optimized formulation was compared with a commercial reference product using similarity factor $\left(f_{2}\right)$ test.

Results: F4 formulation, containing $145 \mathrm{~g}$ of HPMC only, (with $f_{2}$ value of 67.38) was selected as optimized formulation (compared to the reference commercial product), and it released $97.84 \%$ of the drug in $12 \mathrm{~h}$. The release data showed best fit to first-order kinetics $\left(R^{2}=0.9963-0.9989\right)$, though nonsignificantly $(p>0.05)$ different from the Higuchi model $\left(R^{2}=0.9813-0.9955\right)$ except for formulation F6. Based on Koppcha model data, drug release mechanism involved both diffusion and erosion $(n=0.513$ - 0.597) with diffusion being dominant. The optimized formulation exhibited $162 \%$ swelling at the end of $11 \mathrm{~h}$, after which no further weight gain occurred.

Conclusion: Suitable sustained-release tablets of tizanidine hydrochloride have been successfully prepared using direct compression Drug release is sustained by increasing the content of the matrix polymers used.
\end{abstract}

Keywords: Tizanidine, HPMC, EC, Koppcha, Hixson-Crowell, Stokes-Einstein's equation

Tropical Journal of Pharmaceutical Research is indexed by Science Citation Index (SciSearch), Scopus, International Pharmaceutical Abstract, Chemical Abstracts, Embase, Index Copernicus, EBSCO, African Index Medicus, JournalSeek, Journal Citation Reports/Science Edition, Directory of Open Access Journals (DOAJ), African Journal Online, Bioline International, Open-J-Gate and Pharmacy Abstracts

\section{INTRODUCTION}

Tizanidine hydrochloride (THC) is an $\mathrm{a}_{2-}$ adrenergic receptor agonist. It decreases pathologically increased painful spasticity by increasing pre-synaptic inhibition of motor neurons. Plasma protein binding of THC is about $30 \%$ with an elimination $t_{1 / 2}$ of $2-4 h$ [1].

Direct compression method is a less complex methods for temporal drug delivery to the systemic circulation due to easy manufacturing process, more suitability for moisture- and heat- 
sensitive drugs, less likelihood of wear and tear of punches, and more stability of active pharmaceutical ingredients (APIs) [2].

Hydroxypropylmethylcellulose (HPMC) is a semisynthetic derivative of cellulose with ether. It can get easily dissolved in water at all $\mathrm{pH}$ values. It is a good compressible powder and thus provides an opportunity for direct compression. The polymer is thought to be an important hydrophilic carrier employed for preparing oral SR dosage forms $[3,4]$. Ethylcellulose (EC), an ethyl ether of cellulose, is a long chain polymer of $\beta$ anhydroglucose units that are joined together by $1 \rightarrow 4$ acetal linkage [5]. Being hydrophobic and non-biodegradable in nature, EC releases the drug both by diffusion and erosion mechanism. Drug-polymer ratio, polymer concentration and viscosity grade influence the drug release profile from the EC matrix system [6]. EC is effectively employed for the purpose of formulating SR dosage forms. EC coating has been employed in colonic targeted drug delivery system (DDS), along with pectin [6] and amylose [7] that are subjected to enzymatic degradation in colon, thus releasing the drug in colonic region in a controlled manner $[8,9]$.

The aim of this work was to design SR matrix tablets of THC and to look into the effect of HPMC and EC on the drug release profile of THC. Matrix tablets were prepared by directly compressing a blend of drug, polymers (HPMC and/or EC) and excipients.

\section{EXPERIMENTAL}

\section{Materials}

Materials used in this experimental study include: Pure THC powder donated by Stand-Pharma, Lahore, Pakistan. HPMC K100 and EC $10 \mathrm{cps}$ was supplied by International Laboratories
Corporation, USA. Microcrystalline cellulose as Avicel ${ }^{\circ} \mathrm{PH} 101$, talc and magnesium stearate was acquired from Merck Chemicals, Germany. All other chemicals used in this experimental study were of analytical grade.

\section{Preparation of THC matrix tablets}

Matrix tablets were prepared by employing direct compression technique as per formulations mentioned in Table 1.

In all formulations, amount of THC was $6.87 \mathrm{mg}$ (equivalent to $6 \mathrm{mg}$ pure base) and the tablets were having a total weight $300 \mathrm{mg}$. The drug, Avicel $\AA \mathrm{PH} 101$, polymers, magnesium stearate and talc were screened through 60 mesh sieve. All the ingredients were weighed in a precise manner. The ingredients were subjected to mixing for a period of $10 \mathrm{~min}$. In final stage, magnesium stearate was added and the mixture was mixed for further $5 \mathrm{~min}$. Then the powder mix was subjected to compression process using $10.3 \mathrm{~mm}$ single concave surface punch (Emmay, Lahore, Pakistan) [2].

\section{Physical characterization of THC tablets}

The prepared tablets were assessed for weight variation, content uniformity, and friability. These tablets were also subjected to measurement of hardness, thickness and diameter. Weight variation test was performed for 10 tablets from all batches using an electronic balance (model ATY 224, Shimadzu, USA) and average values were calculated [2].

For hardness test, 10 tablets were chosen from each batch and were subjected to hardness test, by means of hardness tester (Emmay, Lahore, Pakistan) and average values were calculated. For determination of content uniformity, 10 tablets were selected and subjected to trituration in a mortar and pestle; and the quantity of ground

Table 1: Composition of tizanidine hydrochloride matrix tablets

\begin{tabular}{lccccccc}
\hline Code & \multicolumn{7}{c}{ Ingredient per tablet (mg) } \\
\cline { 2 - 8 } & HPMC & EC & $\begin{array}{c}\text { Avicel PH } \\
\mathbf{1 0 1}\end{array}$ & Drug & Talc & Magnesium stearate & Total \\
\hline F1 & 290 & 0 & 0 & 6.87 & 2 & 1.13 & 300 \\
F2 & 0 & 290 & 0 & 6.87 & 2 & 1.13 & 300 \\
F3 & 145 & 72.5 & 72.5 & 6.87 & 2 & 1.13 & 300 \\
F4 & 145 & 0 & 145 & 6.87 & 2 & 1.13 & 300 \\
F5 & 145 & 145 & 0 & 6.87 & 2 & 1.13 & 300 \\
F6 & 0 & 145 & 145 & 6.87 & 2 & 1.13 & 300 \\
F7 & 72.5 & 145 & 72.5 & 6.87 & 2 & 1.13 & 300 \\
\hline
\end{tabular}


powder equivalent to single dose of THC was dissolved in $0.1 \mathrm{~N} \mathrm{HCl}$ solution by sonication for a period of $30 \mathrm{~min}$. The drug content was assayed at $\lambda \max 319 \mathrm{~nm}$ using a UV spectrophotometer (UV-3000, O.R.I, Germany). For friability, 10 tablets were taken from each batch, de-dusted and placed in friabilator (Emmay, Lahore, Pakistan) and were rotated at $25 \mathrm{rpm}$ for $4 \mathrm{~min}$. The percent weight loss was calculated for each batch. Diameter as well as thickness of 10 units was measured form each batch and mean values were determined [1].

\section{In vitro dissolution study}

The dissolution study was performed in USP basket dissolution apparatus (apparatus I) at a speed of $100 \mathrm{rpm}$ [1]. The dissolution medium was $0.1 \mathrm{~N} \mathrm{HCl}$ solution (for the initial $2 \mathrm{~h}$ ) and phosphate buffer of $\mathrm{pH} 6.8$ (for remaining $10 \mathrm{~h}$ ). Samples $(5 \mathrm{ml})$ were drawn at predetermined time intervals $(0,1.0,2.0,3.0,4.0,5.0,6.0,7.0$, 8.0, 9.0, 10.0, 11.0, and12.0 h), filtered and analyzed using a UV spectrophotometer (UV3000 , O.R.I, Germany) at $\lambda_{\max }$ of $319 \mathrm{~nm}$ [1]. The sampling volume was replaced by dissolution medium $(5 \mathrm{ml})$. The quantity of drug released in term of percent was determined for all formulations.

\section{Drug release kinetics using model dependent approaches}

DD Solver® software was used for the quantification of the following kinetic models [10]: zero-order, first-order and Higuchi models [1117] as shown in Eqs 1 - 3, respectively.

$M_{t}=M_{o}-K_{o} t$

$\ln \mathrm{M}_{\mathrm{t}}=\ln \mathrm{M}_{\mathrm{o}}-\mathrm{K}_{1} \mathrm{t}$

$Q_{t}=K_{H} t^{1 / 2}$

where $M_{t}=$ quantity of drug remaining undissolved at time ( $\mathrm{t}), \mathrm{M}_{\mathrm{o}}=$ quantity of drug remaining un-dissolved at time $(t)=0, t=$ time of sampling, $Q_{t}=$ quantity of drug remaining undissolved at time $(\mathrm{t})$, and $\mathrm{K}_{\mathrm{o}}, \mathrm{K}_{1}$ and $\mathrm{K}_{\mathrm{H}}$ are the release rate constant for zero-order, first-order and Higuchi models, respectively. Zero-order model shows same quantity of drug is released in different units of time of same duration. Pharmaceutical dosage forms that follows firstorder kinetics, release the quantity of drug that is directly proportional to the quantity of drug remaining in its inner side. Higuchi describes that the drug release occurs through diffusion process that is based on Fick's law of diffusion and is inversely related to square-root of time.
The data were also subjected to Hixson-Crowell model (Eq 4) to ascertain the change in surface area and shape of tablet during the time course of dissolution [18].

$W_{o}^{1 / 3}-W_{t}^{1 / 3}=K_{s} t$

where $\mathrm{Wt}=$ quantity of drug un-dissolved at time $(\mathrm{t}), \mathrm{W}_{\mathrm{o}}=$ quantity of drug un-dissolved at time $(\mathrm{t})$ $=0$ and $\mathrm{K}_{\mathrm{s}}$ is the constant showing the relationship between surface and volume.

For the evaluation of mechanism of drug release, the drug release data were fitted to KorsmeyerPeppas equation (Eq 5) [17].

$\log \left[M_{t} / M_{f}\right]=\log k+n \log t$

where $M_{t}=$ quantity of drug release at time $(t), M_{f}$ $=$ quantity of drug release at infinite time, $\mathrm{k}$ is the release rate constant and $n=$ release exponent that indicates the drug release mechanism. For a cylindrical shape matrix, the value of $n \leq 0.45$ represents Fickian release; $n$ greater than 0.45 but lesser than 0.89 indicates anomalous (nonFickian) release; and $\mathrm{n}$ greater than 0.89 represents super case-II type release.

To ascertain the dominant mechanism between diffusion and erosion, the release data were analyzed using Koppcha model (Eq 6) [18].

$M=A t^{1 / 2}+B$

where $\mathrm{M}=$ cumulative percent of drug released at time $(\mathrm{t}), \mathrm{A}$ and $\mathrm{B}$ are diffusion and erosion terms, respectively. In Koppcha equation, if $A / B \geq$ 1 , then the process of difusion predominates, while if $A / B<1$, then the process of erosion prevails.

\section{Evaluation of similarity factor $\left(f_{2}\right)$}

Finally, all the formulations were compared with the marketed formulation based on similarity factor $\left(f_{2}\right)$ as in Eq 7 .

$f 2=50 X \log \left[1+\left(\frac{1}{n}\right) \sum_{t=1}^{n}\left(R_{l}-T_{i}\right)^{2}\right] \times 100$

where $n$ is sampling number, $R_{t}$ is percent of drug dissolved of the reference product and $T_{t}$ is percent of drug dissolved of test products. For similar release profiles, similarity factor should be in the range of 50 - 100 (closer to 100) [3].

\section{Swelling studies}

The degree of swelling was assessed from the percentage weight gain/water uptake by the tablets. For this purpose, six tablet from the 
optimum formulation was placed in a petri-dish containing $40 \mathrm{ml}$ of phosphate buffer $(\mathrm{pH}=6.8)$ for $1 \mathrm{~h}$, after which the tablets were taken, dried in oven in order to remove excess of phosphate buffer and weighed. Thereafter, the tablets were placed back in the buffer and weighed at every 2 $\mathrm{h}$ interval after blotting out excess fluid, and the process was continued over a period of $12 \mathrm{~h}$. Swelling index (\%) was calculated by using Eq 8 [8].

SI $(\%)=\left\{\left(M_{t}-M_{0}\right) / M_{o}\right\} 100$

where $\mathrm{SI}$ is swelling index, $\mathrm{M}_{\mathrm{t}}$ is the weight of the tablets at time (t) and $M_{0}$ is the initial weight of the tablets.

\section{Assessment of in vitro buoyancy of tablets}

In vitro buoyancy was determined from floating lag-time as described previously [5]. The tablet was kept in a beaker $(100 \mathrm{ml})$ containing $0.1 \mathrm{M}$ $\mathrm{HCl}$. The time taken before the tablet rose to the surface of the liquid was considered as floating lag-time.

\section{Statistical analysis}

All the data were analyzed using Microsoft Excel®, version 17.0. One-way ANOVA was

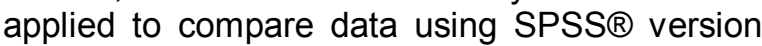
16.0 software. The level of significance was set at $p<0.05$.

\section{RESULTS}

In formulation F6, F7 and F5 (Figure 1), the release retardant effect increased with increasing amount of HPMC in formulations. The $Q_{2}$ values for formulations F6, F7 and F5 are $101 \%, 45.83$ $\%$ and $42.54 \%$, respectively. There is a significant $(p>0.05)$ difference between these values. The $Q_{6}$ values for formulations $F 7$ and F5 are $76.93 \%$ and $71.58 \%$, respectively which are significantly $(p>0.05)$ different from each other. The $Q_{12}$ values for formulations $F 7$ and $F 5$ are $95.11 \%$ and $92.52 \%$, respectively. There is a significant $(p>0.05)$ difference between these values. Moreover, $\mathrm{Q}_{2}$ is amount of drug released after $2 \mathrm{~h}, \mathrm{Q}_{6}$ is amount of drug released after $6 \mathrm{~h}$ and $Q_{12}$ is amount of drug released after $12 \mathrm{~h}$.

For formulations F4, F3 and F5 (Figure 2), the $\mathrm{Q}_{2}$ values are 46.18, 44.4 and $42.54 \%$, respectively. There is a significant $(p>0.05)$ difference between these values. $Q_{6}$ values for formulations F4, F3 and F5 are 80.99, 76.08 and $71.58 \%$, respectively exhibiting significant $(p>$ $0.05)$ difference between these values. $Q_{12}$ values for formulations $\mathrm{F} 4, \mathrm{~F} 3$ and $\mathrm{F} 5$ are 97.84 $\%, 95.53 \%$ and $92.52 \%$, respectively. There is a significant $(p>0.05)$ difference between these values. The drug release from $F 1\left(Q_{2}=38.25 \%\right.$, $\mathrm{Q}_{6}=66.88 \%$ and $\mathrm{Q}_{12}=85.52 \%$ ) was less than that of $F 4\left(Q_{2}=46.18 \%, Q_{6}=80.99 \%\right.$ and $Q_{12}$ $=97.84 \%)$ at specific time points and this difference was statistically significant $(p<0.05)$ (Figure 3). The drug release from $\mathrm{F} 2\left(\mathrm{Q}_{2}=18.34\right.$ $\%, Q_{6}=28.18 \%$ and $Q_{12}=35.24 \%$ ) was less than that of $F 6\left(Q_{2}=101.07 \%\right)$ at specific time points and this difference was statistically significant ( $p$ $<0.05$ ) (Figure 3).

Based on similarity factor, F4 was selected as optimum formulation. F4 has a similarity factor of 67.38 , followed by $\mathrm{F} 7\left(f_{2}=64.03\right)$. F3 and F5 has similarity factor of 60.36 and 52.01 , respectively. All other formulations have similarity factors below 50 (42.39 for F1, 14.06 for F2 and 27.20 for F6).

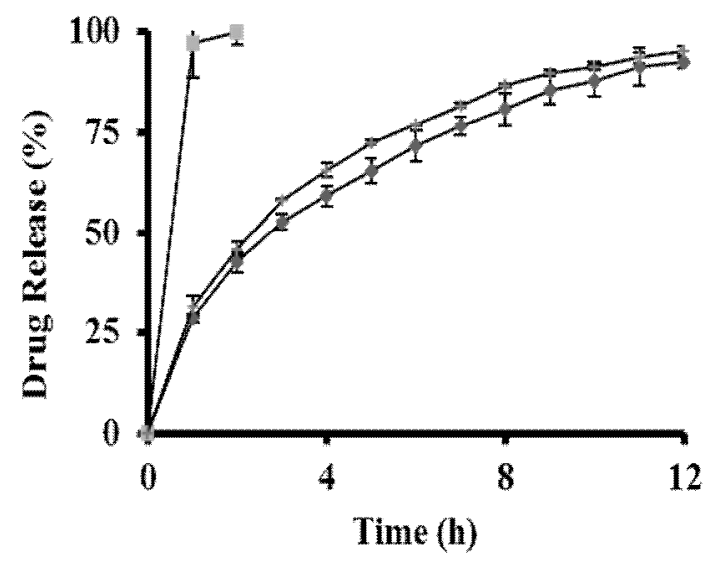

Figure 1: Influence of HPMC on release of drug; mean $\pm S D(n=3)(\diamond F 5$, - F6, + F7)

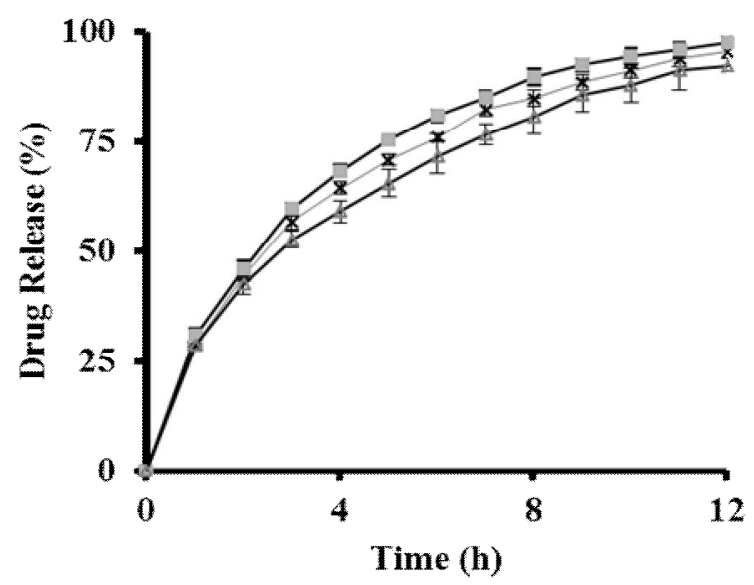

Figure 2: Influence of EC on release of drug; mean \pm $\mathrm{SD}(\mathrm{n}=3)(\times \mathrm{F} 3, \mathbf{F} 4, \Delta \mathrm{F} 5)$

Trop J Pharm Res, February 2015; 14(2): 222 


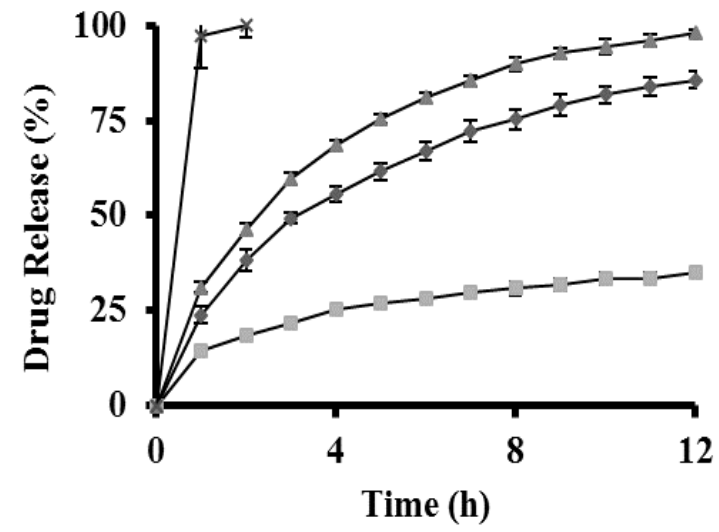

Figure 3: Influence of Avicel $^{\circledR} \mathrm{PH} 101$ on release of drug; mean $\pm \mathrm{SD}(\mathrm{n}=3)(\diamond \mathrm{F} 1, \boldsymbol{\bullet} \mathrm{F} 2, \boldsymbol{\Delta} \mathrm{F} 4, \times \mathrm{F} 6)$

Except F2, all the formulations followed firstorder drug release kinetics where the $R^{2}$ values were in the range of $0.9963-0.9989$, however $R^{2}$ values for first order equation are nonsignificantly $(p>0.05)$ different from that of Higuchi model i.e. $R^{2}=0.9813-0.9955$ except formulation F6.

The optimum formulation (F4) successively gained at a maximum weight (161.99\%) up to 11 $\mathrm{h}$ and underwent swelling. But after that the weight was again decreased.

\section{DISCUSSION}

To imitate the effect of variable $\mathrm{pH}$ of GIT, dissolution studies of SR tablets of all formulations were carried out at the most common extremes of $\mathrm{pH}$, as $\mathrm{pH}$ of GIT lies in this range of $\mathrm{pH} 1.2$ and $\mathrm{pH} 6.8$.

By incorporating HPMC to the matrix, release (Figure 1) of drug is retarded due to formation of gel barrier which hinders the dissolution of drug as it does not allow dissolution medium to get infiltrated to the core of the matrix to dissolve the drug.

In this study, SR formulation of THC was developed by employing hydrophilic polymer (HPMC) in combination with hydrophobic polymer (EC). Other excipients like talc and magnesium stearate were kept in a constant amount in order to determine the influence of HPMC in a clear way. F6 quickly disintegrated due to hydrophilic nature of Avicel $\circledast$ PH 101 and did not give any sustaining effect. The release retardant effect was increased with increase in the amount of HPMC as expected. This increased release retardant effect of HPMC may be due to the formation of gel barrier that hinders the penetration of dissolution medium to the matrix. It may also be due to slow erosion of the matrix. As reported previously [2], the most important factor that affects the drug release from HPMC matrix is drug to polymer ratio. Increasing polymer concentration in the matrix leads to boost the viscosity of gel, which in turn may lead to a decrease in diffusion coefficient of drug and thereby reducing the drug release.

Table 2: Drug release kinetics of tizanidine hydrochloride matrix tablets

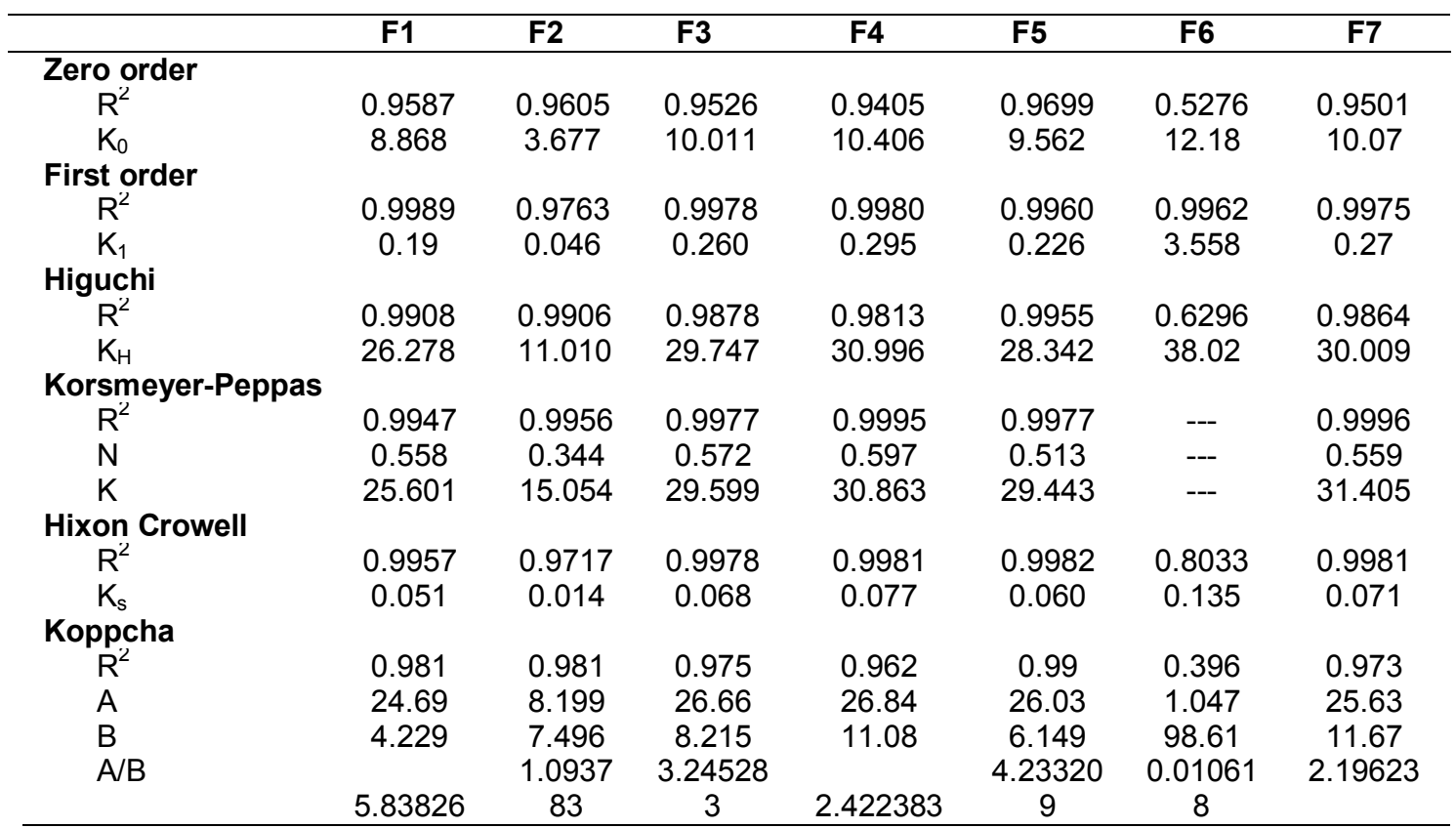


In present study, it was noticed that by increasing concentration of HPMC the drug release retardant effect was increased and vice versa. It is recommended that the polymer like HPMC having hydrophilic characteristic when encounter water, absorbs it and undergoes swelling thus forming a gel layer that serves as a blockade to the process of diffusion. The course of drug release from hydrophilic matrix involves penetration of water into the matrix system, hydration followed by gel formation, drug dissolution and diffusion of drug through the resultant gel barrier.

As the hydrophilic HPMC quickly undergoes hydration on the outermost surface of tablet, thus forming a gel barrier, which acts as a physical and diffusion barrier thus retarding the drug release by diffusion [9]. This gel barrier also prevents wetting of the core and hinders the tablet to undergo disintegration [15]. It is also suggested that the gel barrier with higher viscosity of HPMC resulted in a more sluggish release of metoprolol due to formation of barrier to that is more tortuous and resistant diffusion [6].

This release retardant effect of EC is most likely due to its hydrophobic character that hinders the infiltration of dissolution medium into the matrix thus retarding the dissolution and subsequent release of the drug. For the purpose of studying the influence of EC on drug release, formulations F4, F3 and F5 were evaluated. There was a regular but not very proportional decrease in drug release among these three formulations. The decrease in drug release with increasing EC contents are in accord with previous result [7] who used water insoluble polymer for reducing drug release in combination with HPMC.

Moreover, formulation F2 sustained the drug release more pronouncedly than the other formulations while diffusion controlled release of drug from formulation F2 was observed. The drug release kinetics followed Higuchi's model. This may be attributed to sluggish process of matrix hydration, due to the hydrophobic nature of EC, whereby the insoluble particles can act as barrier to drug release due to formation of a tortuous matrix having diminished porosity and thus the drug release from matrix is dependent upon the square root of time process based on Fickian diffusion. Also, hydrophobic polymers limit the infiltration of medium in to the matrix as well as confine the development of gel layer on the outer surface of matrix as compared to the hydrophilic HPMC [9].
Except F2, all the formulations followed firstorder drug release kinetics where the $R^{2}$ values were in the range of 0.9963-0.9989, however $R^{2}$ values for first order equation are nonsignificantly $(p>0.05)$ different from that of Higuchi model i.e. $\mathrm{R}^{2}=0.9813-0.9955$ except formulation $\mathrm{F} 6$. This release pattern may due to change in surface area of the tablets as both diffusion as well as erosion is involved in dissolution process of these formulations. Another possible reason could be the initial dissolution of formulations in $0.1 \mathrm{~N} \mathrm{HCl}$ solution in which comparatively swift release of drug occurred from the tablets as compared to that in phosphate buffer $\mathrm{pH} 6.8$. The drug release data also fit to Hixson-Crowell cube-root law that demonstrates the drug release from systems, which involve a change in surface area and diameter of the particles or tablets [15]. In case of Korsmeyer-Peppas equation, F2 follows mechanism of diffusion for drug release $(n=$ 0.344), whereas formulations F1, F3, F4, F5 and F7 underwent both diffusion and erosion ( $\mathrm{n}=$ 0.513-0.597). For the evaluation of predominant mechanism between diffusion and erosion, dissolution data was treated with Koppcha equation (equation 6 ). In this equation, if $A / B \geq 1$, then the process of diffusion predominates, while for $A / B<1$, erosion prevails. These values are presented in Table 2. In formulations F1, F3, F4, $\mathrm{F} 5$ and $\mathrm{F} 7$, whereby both diffusion and erosion were present, diffusion predominates over erosion as value of $A$ is greater than that of $B$ in this case [17]. Furthermore, F6 disintegrated immediately and did not fulfill the criteria for sustained release dosage form due to the presence of hydrophilic Avicel ${ }^{\circ} \mathrm{PH} 101$, which infiltrates the dissolution medium into the matrix leading to disintegration.

The swelling behavior and in vitro buoyancy of the optimum formulation (F4; containing HPMC but not EC) were studied in term of percent weight gain and floating lag-time, respectively. The optimum formulation (F4) successively gained at a maximum weight $(161.99 \%)$ up to 11 h) and underwent swelling. But after that the weight was again decreased. This is due to penetration of water in the glassy network of polymer. Due to the presence of water, the glass transition temperature of the matrix is decreased to that of the dissolution medium and a transition from glassy to rubbery states takes place as the amount of water inside the matrix increases. The intake of water leads to induction of stress within the polymeric matrix. Consequently, the polymeric matrix relaxes and swelling occurs [8]. 
Furthermore, the optimum formulation (F4) did not float up to $17 \mathrm{~min}$.

\section{CONCLUSION}

Tizanidine hydrochloride SR tablets can be suitably prepared by direct compression using a combination of hydrophilic and hydrophobic polymers. Both HPMC and EC are capable of retarding drug release either alone or in combination, and there is a direct relation between the concentration of polymer and the release-retardant effect of the two polymers. Drug release decreases with increasing concentration of the polymers in the matrix. Tizanidine hydrochloride is released by a combined diffusion/erosion mechanism from the formulations.

\section{REFERENCES}

1. Khan SA, Ahmad M, Murtaza G, Aamir MN, Madni MA, Kousar R, Asghar MW. Formulation of two-drug controlled release non-biodegradable microparticles for potential treatment of muscles pain and spasm and their simultaneous spectrophotometeric estimation. Acta Pol. Pharm. Drug Res. 2010; 67(3): 299-306.

2. Baseer A, Hassan F, Hassan SMF, Jabeen S, Israr F, Murtaza G, Haque N. Physico-chemical comparison of famotidine tablets prepared via dry granulation and direct compression techniques. Pak. J. Pharm. Sci. 2013; 26(3): 439-443.

3. Shahzad MK, Ubaid, M, Raza, M, Murtaza G. The formulation of flurbiprofen loaded microspheres using hydroxypropylmethylcellulose and ethylcellulose. Adv. Clin. Exp. Med. 2013; 22(2): 177-183.

4. Khan SA, Ahmad M, Murtaza G, Muhammad NA, Nisar UR, Rozina K, Fatima R, Mohammad A. Formulation of nimesulide floating microparticles using low viscosity hydroxypropyl mehylcellulose. Trop. J. Pharm. Res. 2010; 9(2): 293-299.

5. Murtaza G. Ethylcellulose microparticles: A review. Acta Pol. Pharm. Drug Res. 2012; 69(1): 11-22.

6. Murtaza G, Ahmad M, Akhtar N. Biowaiver study of oral tabletted ethylcellulose microcapsules of a BCS class I drug. Bull. Chem. Soc. Ethiop. 2009; 23(1): 1-16.

7. Murtaza G, Ahmad M, Asghar MW, Aamir MN. Salbutamol sulphate-ethylcellulose microparticles: formulation and in-vitro evaluation with emphasis on mathematical approaches. DARU J. Pharm. Sci. 2009; 17(4): 209-216.

8. Murtaza G, Ahmad M, Shehnaz G. Microencapsulation of diclofenac sodium by non-solvent addition technique: Use of toluene and petroleum benzin as solvent and non-solvent respectively. Trop. J. Pharm. Res. 2010; 9(1): 187-195.

9. Murtaza G, Ahamd M, Akhtar N, Rasool F. A comparative study of various microencapsulation techniques: Effect of polymer viscosity on microcapsule characteristics. Pak. J. Pharm. Sci. 2009; 22(5): 291 300.

10. Murtaza G. Development of glutaraldehyde cross-linked metronidazole loaded chitosan microcapsules: analysis of dissolution data using $D D$-Solver. Latin Am. J. Pharm. 2011; 30(2): 301-308.

11. Rasool R, Ahmad M, Murtaza G, Khan HMS, Khan SA. Comparative dissolution study of metoprolol tartrate loaded PLGA (50:50) and PLGA (75:25) microparticles. Asian J. Chem. 2011; 23(4): 38153818.

12. Rasool F, Ahmad M, Khan HMS, Akhtar N, Murtaza G. The effect of binders on the bioavailability of ofloxacin tablets in animal model. Acta Pol. Pharm. Drug Res. 2010; 67(1): 185-189.

13. Khan SA, Ahmad M, Murtaza G, Shehnaz G, Aamir MN, Rasool F. Design of nimesulide-chitosan microparticles by $\mathrm{pH}$ change coacervation. Latin. Am. J. Pharm. 2011; 30(6): 1260-1266.

14. Khan SA, Ahmad M, Kousar R, Murtaza G. Nimesulideserratiopeptidase sustained release microparticlesCombined formulation and in vitro characterization. Adv. Clin. Exp. Med. 2011; 20(2): 605-611.

15. Aamir MN, Ahmad M, Akhtar N, Murtaza G, Khan SA, Zaman SU, Nokhodchi A. Development and in vitro-in vivo relationship of controlled-release microparticles loaded with tramadol hydrochloride. Int. J. Pharm. 2011; 407: 38-43.

16. Aamir MN, Ahmad M, Khan SA, Akhtar N, Murtaza G, Akhtar M, Rasool F. Fabrication of novel gastroretentive floating microcapsules by utilizing combination of HPMC and EC and their Physicochemical Assessment. Latin Am. J. Pharm. 2011; 30(5): 1010-1015.

17. Aamir MN, Ahmad M, Murtaza G, Akhtar N, Khan SA, Usman M. Synthesis of biodegradable microspheres of tramadol by simple phase separation technique and their in vitro evaluation. Lat. Am. J. Pharm., 2010; 29(1): 1152-1158.

18. Kopcha M, Lordi NG, Tojo KJ. Evaluation of release from selected thermosoftening vehicles. J. Pharm. Pharmacol. 1991; 43(6): 382-387. 